\title{
A GENERALISATION OF UNIFORM MATROIDS
}

\author{
GEORGE DRUMMOND
}

\begin{abstract}
A matroid is uniform if and only if it has no minor isomorphic to $U_{1,1} \oplus U_{0,1}$ and is paving if and only if it has no minor isomorphic to $U_{2,2} \oplus U_{0,1}$. This paper considers, more generally, when a matroid $M$ has no $U_{k, k} \oplus U_{0, \ell}$-minor for a fixed pair of positive integers $(k, \ell)$. Calling such a matroid $(k, \ell)$-uniform, it is shown that this is equivalent to the condition that every rank- $(r(M)-k)$ flat of $M$ has nullity less than $\ell$. Generalising a result of Rajpal, we prove that for any pair $(k, \ell)$ of positive integers and prime power $q$, only finitely many simple cosimple $G F(q)$-representable matroids are $(k, \ell)$-uniform. Consequently, if Rota's Conjecture holds, then for every prime power $q$, there exists a pair $\left(k_{q}, \ell_{q}\right)$ of positive integers such that every excluded minor of $G F(q)$-representability is $\left(k_{q}, \ell_{q}\right)$-uniform. We also determine all binary $(2,2)$-uniform matroids and show the maximally 3 -connected members to be $Z_{5} \backslash t, A G(4,2), A G(4,2)^{*}$ and a particular self-dual matroid $P_{10}$. Combined with results of Acketa and Rajpal, this completes the list of binary $(k, \ell)$-uniform matroids for which $k+\ell \leq 4$.
\end{abstract}

\section{INTRODUCTION}

A matroid $M$ is paving if every rank- $(r(M)-2)$ flat is independent, or equivalently, if $M \mid H$ is uniform for every hyperplane $H$ of $M$. Thus, in a natural sense, paving matroids are close to uniform. In this paper, we generalise this observation and describe a two-parameter property of matroids that captures just how close to uniform a given matroid is.

For positive integers $k$ and $\ell$, we define a matroid to be $(k, \ell)$-uniform if it has no minor isomorphic to $U_{k, k} \oplus U_{0, \ell}$. It is easy to show that a matroid is $(1,1)$-uniform precisely if it is uniform and is $(2,1)$-uniform precisely if it is paving. It is also evident that all matroids are $(k, \ell)$-uniform for some $(k, \ell)$ pair and that if $M$ is $(k, \ell)$-uniform, then it is $\left(k^{\prime}, \ell^{\prime}\right)$-uniform for all $k^{\prime} \geq k$ and $\ell^{\prime} \geq \ell$. Furthermore, an easy duality argument shows that a matroid is $(k, \ell)$-uniform if and only if its dual is $(\ell, k)$-uniform. We will use all these facts freely. The first main result of this paper, proved in Section 2, concerns representability of $(k, \ell)$-uniform matroids.

Theorem 1.1. For every pair $(k, \ell)$ of positive integers and every prime power $q$, only finitely many simple cosimple $G F(q)$-representable matroids are $(k, \ell)$-uniform.

2020 Mathematics Subject Classification. 05B35.

Key words and phrases. matroids, uniform, paving, flats, binary. 
Note that both the simple and cosimple requirements in this theorem are necessary, as the uniform matroids $U_{1, n}$ and $U_{n-1, n}$ are representable over every field for all $n \geq 1$. The following is an interesting corollary of this theorem:

Corollary 1.2. For every prime power $q$, the set of excluded minors for $G F(q)$-representability is finite if and only if for some fixed pair $\left(k_{q}, \ell_{q}\right)$ of positive integers, every such excluded minor is $\left(k_{q}, \ell_{q}\right)$-uniform.

To illustrate, for $q \leq 4$, every excluded minor of $G F(q)$-representability is $(2,1)$-uniform, that is, paving. As Geelen, Gerards and Whittle have announced a proof of Rota's Conjecture [2], it would seem that such $\left(k_{q}, \ell_{q}\right)$ pairs exist for all $q$. If well behaved, these bounds may offer improved methods for explicitly determining the excluded minors of $G F(q)$-representability.

By applying duality to the lists of binary $(2,1)$-uniform and $(3,1)$-uniform matroids of Acketa [1] and Rajpal [6] respectively, one may explicitly list all binary (1,2)-uniform and (1,3)-uniform matroids. These results concern binary $(k, \ell)$-uniform matroids such that $k+\ell \leq 4$. We complete this picture in Sections 3 and 4 by determining the binary $(2,2)$-uniform matroids. The most difficult part of the characterisation is in establishing the following result, the proof of which appears in Section 4:

Theorem 1.3. The 3-connected binary $(2,2)$-uniform matroids are precisely the 3 -connected minors of $Z_{5} \backslash t, P_{10}, A G(4,2)$, and $A G(4,2)^{*}$.

Here, $Z_{5} \backslash t$ is the tipless binary 5-spike, $A G(4,2)$ is the rank-5 affine geometry, and $P_{10}$ is the rank- 5 binary matroid represented by the matrix of Figure 1. It is easily seen that $P_{10}$ is self-dual and that $P_{10} / 5 \backslash 10 \cong M\left(\mathcal{W}_{4}\right)$. Moreover, by pivoting, one can show that $P_{10} / 8 \cong Z_{4}$. A further description of $P_{10}$ is given in Section 4. The terminology throughout will follow Oxley [5] unless otherwise specified.

$$
\begin{aligned}
& \begin{array}{llllllllll}
1 & 2 & 3 & 4 & 5 & 6 & 7 & 8 & 9 & 10
\end{array} \\
& {\left[\begin{array}{llllllllll}
1 & 0 & 0 & 0 & 0 & 1 & 0 & 0 & 1 & 1 \\
0 & 1 & 0 & 0 & 0 & 1 & 1 & 0 & 0 & 1 \\
0 & 0 & 1 & 0 & 0 & 0 & 1 & 1 & 0 & 1 \\
0 & 0 & 0 & 1 & 0 & 0 & 0 & 1 & 1 & 0 \\
0 & 0 & 0 & 0 & 1 & 1 & 1 & 1 & 0 & 0
\end{array}\right]}
\end{aligned}
$$

Figure 1. A binary representation of $P_{10}$.

The nullity of a set $X$ in a matroid $M$ is $|X|-r_{M}(X)$. We conclude this section by proving a characterisation of $(k, \ell)$-uniform matroids in terms of nullity of flats that will be treated as an alternate definition.

Proposition 1.4. A matroid $M$ is $(k, \ell)$-uniform if and only if every rank$(r(M)-k)$ flat of $M$ has nullity less than $\ell$. 
Proof. Suppose first that $M$ is not $(k, \ell)$-uniform. Then $M$ has an independent set $X$ and coindependent set $Y$ such that $M / X \backslash Y \cong U_{k, k} \oplus U_{0, \ell}$ and $r_{M}(X)=r(M)-k$. Letting $Z$ denote the $\ell$ loops of $M / X \backslash Y$, every element of $Z$ must be in the closure of $X$ in $M$. Thus, $\operatorname{cl}_{M}(X)$ is a rank- $(r(M)-k)$ flat of $M$ with nullity at least $\ell$.

For the converse, suppose $M$ has a rank- $(r(M)-k)$ flat $F$ of nullity at least $\ell$. Contracting any basis for $F$ achieves a rank- $k$ matroid with at least $\ell$ loops. An appropriate restriction then yields a $U_{k, k} \oplus U_{0, \ell}$-minor.

\section{Finiteness over $G F(q)$}

In this section, we prove Theorem 1.1 by showing that, for any $(k, \ell)$ pair and prime power $q$, there exists a constant $f(k, \ell, q)$ bounding the rank of any simple cosimple $(k, \ell)$-uniform $G F(q)$-representable matroid. We will require the following result, a rewording of [6, Proposition 8].

Lemma 2.1. For $k \geq 2$, let $M$ be a simple $(k, 1)$-uniform matroid such that $M$ is $G F(q)$-representable and $r^{*}(M)>q$. Then there is a constant $g(k, q)$ such that $r(M) \leq g(k, q)$.

We will also make use of the following easy lemma.

Lemma 2.2. If $M$ is a matroid of rank $r \geq 2$, then $M$ is simple if and only if it is $(r-1,1)$-uniform.

Theorem 1.1 is then a direct consequence of the following:

Proposition 2.3. For every pair $(k, \ell)$ of positive integers and every prime power $q$, there is a constant $f(k, \ell, q)$ such that if $M$ is a simple cosimple $G F(q)$-representable $(k, \ell)$-uniform matroid, then $r(M) \leq f(k, \ell, q)$. Moreover, if $k \geq 2$, then

$$
f(k, \ell, q) \leq \max \{f(k-1, \ell+1, q), f(1, \ell, q)+(k-1)\} .
$$

Proof. Fixing $q$, we perform induction on $k$. The base case $k=1$ we split into two parts. Firstly, if $k=\ell=1$, then $M$ is uniform and $r(M) \leq q-1$, as otherwise $M$ has a $U_{q, q+2}$ minor, a contradiction to $G F(q)$-representability. If $k=1$ and $\ell \geq 2$, then by the dual of Lemma 2.1, either $r(M) \leq q$ or $r^{*}(M) \leq g(\ell, q)$. In the latter case, since $M$ is cosimple, $M^{*}$ is a restriction of $P G(g(\ell, q)-1, q)$. It follows that $r(M) \leq\left(q^{g(\ell, q)}-1\right) /(q-1)-g(\ell, q)$. Thus, $f(1, \ell, q)$ exists for all $\ell \geq 1$.

Now suppose $k \geq 2$ and that $f\left(k^{\prime}, \ell^{\prime}, q\right)$ exists for all $k^{\prime}<k, \ell^{\prime} \geq 1$. If $M$ is also $(k-1, \ell+1)$-uniform, then $r(M) \leq f(k-1, \ell+1, q)$ by induction. Otherwise, $M$ has a rank- $(r(M)-k+1)$ flat $F$ with nullity at least $\ell+1$. As $M$ is $(k, \ell)$-uniform, $M \mid F$ is $(1, \ell)$-uniform. By duality, $(M \mid F)^{*}$ is $(\ell, 1)$ uniform and, as $r^{*}(M \mid F) \geq \ell+1$, it follows Lemma 2.2 that $M \mid F$ is cosimple. Thus, $M \mid F$ is a simple cosimple binary $(1, \ell)$-uniform matroid. By induction, $r(M \mid F) \leq f(1, \ell, q)$ and hence $r(M) \leq f(1, \ell, q)+(k-1)$. We conclude that $f(k, \ell, q)$ exists and $f(k, \ell, q) \leq \max \{f(k-1, \ell+1, q), f(1, \ell, q)+(k-1)\}$. 
The next two results are extracted from [1] and [6] respectively.

Lemma 2.4. $f(2,1,2)=f(1,2,2)=4$.

Lemma 2.5. $f(3,1,2)=5$ and $f(1,3,2)=11$.

It follows Proposition 2.3, that a simple cosimple binary $(2,2)$-uniform matroid has rank at most 11. A more helpful bound, that will be instrumental in our determination of the binary $(2,2)$-uniform matroids is the following:

Lemma 2.6. Let $M$ be a simple cosimple binary matroid. If $M$ is $(2,2)$-uniform, then $\min \left\{r(M), r^{*}(M)\right\} \leq 5$.

Proof. If $M$ is $(1,3)$-uniform, then $M^{*}$ is $(3,1)$-uniform and $r^{*}(M) \leq 5$ by Lemma 2.5. Otherwise, $M$ has a hyperplane $H$ of nullity at least 3 . The matroid $M \mid H$ is simple and (1,2)-uniform. Furthermore, its dual is $(2,1)$ uniform and has rank at least 3 . Thus, by Lemma $2.2, M \mid H$ is cosimple. It follows Lemma 2.4 that $r(M \mid H) \leq 4$ and, consequently, $r(M) \leq 5$.

The remaining two sections will make frequent use of the fact that a matroid $M$ is $(2,2)$-uniform if and only if the union of any pair of circuits of $M$ has rank at least $r(M)-1$.

\section{The $(2,2)$-Uniform MATROIDS THAT ARE NOT 3-CONNECTED}

In this section we describe all $(2,2)$-uniform matroids which are not 3 -connected and explicitly list those that are binary. The following results contain some redundancy but have been chosen for their clarity and to emphasise links to paving matroids. A matroid $M$ is sparse paving if both $M$ and $M^{*}$ are paving, or equivalently, if $M$ is both $(2,1)$ and $(1,2)$-uniform.

Proposition 3.1. Let $M$ be a disconnected matroid. Then $M$ is $(2,2)$-uniform if and only if

(i) $\quad M$ or $M^{*}$ is paving; or

(ii) $\quad M \cong M_{p} \oplus U_{0,1}$ or $M \cong M_{p}^{*} \oplus U_{1,1}$, where $M_{p}$ is a paving matroid; or

(iii) $\quad M \cong M_{p} \oplus U_{1,2}$, where $M_{p}$ is a sparse paving matroid.

Proof. The disconnected matroids of type (i), (ii) and (iii) are easily seen to be $(2,2)$-uniform. To see that there are no others, let $M$ be a disconnected $(2,2)$-uniform matroid. If $M$ has a loop $l$, then $M \backslash l$ is certainly paving and

(ii) holds. Otherwise, by duality, we may assume that $M$ has no loops or coloops. It follows that if $r(M) \leq 2$ or $r^{*}(M) \leq 2$, then (i) holds. Hence, we may also assume that $r(M), r^{*}(M) \geq 3$. Now, if every component of $M$ has rank, corank at least two, then each component contains at least two circuits and the union of any two such circuits has rank less than $r(M)-1$, a contradiction to the $(2,2)$-uniform property. Thus, up to duality, $M$ has at least one rank-1 component $M_{1}$. If $\left|E\left(M_{1}\right)\right| \geq 3$, then by the (2,2)-uniform property, $r(M) \leq 2$, a contradiction. Thus, $M_{1} \cong U_{1,2}$. It then follows easily from the $(2,2)$-uniform property that $M \backslash E\left(M_{1}\right)$ is both $(2,1)$-uniform and $(1,2)$-uniform. In particular, (iii) is satisfied. 
We follow Oxley [5] in using $P\left(M_{1}, M_{2}\right)$ to denote the parallel connection of matroids $M_{1}$ and $M_{2}$ across some common basepoint.

Proposition 3.2. Let $M$ be a connected matroid that is not 3-connected. Then $M$ is $(2,2)$-uniform if and only if

(i) $\quad M$ or $M^{*}$ is paving; or

(ii) $\quad M$ or $M^{*}$ has rank 3 and no parallel class of size more than two; or

(iii) $M$ has a parallel or series pair $\left\{p, p^{\prime}\right\}$ such that $M \backslash p / p^{\prime}$ is sparse paving; or

(iv) $\quad M=P\left(N, U_{2,4}\right) \backslash p$, where $N$ is a connected matroid such that $N / p$ and $N^{*} / p$ are paving.

Proof. It is straightforward to show that all matroids of type (i)-(iv) are $(2,2)$-uniform. To see that this list is complete, let $M$ be a connected $(2,2)$ uniform matroid that is not 3 -connected. If $M$ has rank or corank at most 3 , then it is easily seen to satisfy (i) or (ii). Thus, we may assume that $r(M), r^{*}(M) \geq 4$. Suppose now that, up to duality, $M$ has a parallel pair $\left\{p, p^{\prime}\right\}$ and let $N=M \backslash p / p^{\prime}$. If there exists a circuit $C$ of $N$ of rank at most $r(N)-2$, then as $C$ or $C \cup p^{\prime}$ is a circuit of $M$, it follows that $C \cup\left\{p, p^{\prime}\right\}$ contains two circuits of $M$ whose union has rank at most $r(N)-1=r(M)-2$. Similarly, if there exists a pair of circuits $C_{1}, C_{2}$ of $N$ such that $r_{N}\left(C_{1} \cup C_{2}\right) \leq$ $r(N)-1$, then $C_{1} \cup C_{2} \cup\left\{p, p^{\prime}\right\}$ contains two circuits of $M$ whose union has rank at most $r(M)-2$. Both situations contradict the fact that $M$ is (2,2)-uniform. Hence, $N$ is sparse paving and (iii) holds. Otherwise, $M$ has no parallel or series pairs and we may assume that $M=P\left(M_{1}, M_{2}\right) \backslash p$, for some connected matroids $M_{1}, M_{2}$ each having at least three elements and rank, corank at least two. If $r\left(M_{1}\right), r\left(M_{2}\right) \geq 3$, then by the $(2,2)$ uniform property, each of $M_{1} \backslash p$ and $M_{2} \backslash p$ contains at most one circuit. As each $M_{i}$ is connected, it follows that for $i \in\{1,2\}, M_{i} \backslash p$ is a circuit and $r^{*}(M) \leq 3$, a contradiction. Thus, without loss of generality, $r\left(M_{1}\right)=2$ and $r(M)=r\left(M_{2}\right)+1$. If $\left|E\left(M_{1}\right)\right| \geq 5$, then $E\left(M_{1}\right)-p$ contains two triangles of $M$, and by the $(2,2)$-uniform property, $r(M) \leq 3$, a contradiction. Thus, $M_{1} \cong U_{2,4}$. Now let $T=E\left(M_{1}\right)-p$. By the (2,2)-uniform property, $r_{M}(C \cup T) \geq r(M)-1=r\left(M_{2}\right)$ for every circuit $C$ of $M_{2}$. It follows that every circuit of $M_{2}$ containing $p$ must have rank at least $r\left(M_{2}\right)-1$ and every circuit avoiding $p$ has rank at least $r\left(M_{2}\right)-2$. Thus, $M_{2} / p$ is paving. Also by the (2,2)-uniform property, every pair of circuits of $M_{2} \backslash p$ must span. We conclude that $M_{2} / p$ and $M_{2}^{*} / p=\left(M_{2} \backslash p\right)^{*}$ are paving and that (iv) holds.

Restricting our attention to binary matroids, we may ignore case (iv) of Proposition 3.2 as such matroids have a $U_{2,4}$-minor. We then achieve the following list by combining Propositions 3.1 and 3.2 with Acketa's list [1] of binary paving matroids. Note that, as $M\left(\mathcal{W}_{3}\right), F_{7}, F_{7}^{*}$ and $A G(3,2)$ have transitive automorphism groups, any parallel connections of these matroids and $U_{2,3}$ are free of reference to a specific basepoint. The matroid $S_{8}$ is isomorphic to the unique non-tip deletion of the binary 4-spike $Z_{4}$. 
Corollary 3.3. The following matroids and their duals are all the binary $(2,2)$-uniform matroids that are not 3-connected.

(i) The matroids of rank at most 1 other than $U_{0,1}, U_{1,1}, U_{1,2}, U_{1,3}$;

(ii) the non-simple rank-2 binary matroids with at most one loop;

(iii) the looples, non-simple rank-3 binary matroids with every parallel class of size at most 2 ;

(iv) $M_{p} \oplus U_{0,1}$ and $M_{p} \oplus U_{1,2}$, for $M_{p}$ in $\left\{M\left(\mathcal{W}_{3}\right), F_{7}, F_{7}^{*}, A G(3,2)\right\}$;

(v) $P\left(Z_{4}, U_{2,3}\right) \backslash t$ and $P\left(S_{8}, U_{2,3}\right) \backslash t$, where $t$ is the tip of $Z_{4}$;

(vi) $P\left(F_{7}, U_{2,3}\right) \backslash p$ and $P\left(A G(3,2), U_{2,3}\right) \backslash p$; and

(vii) $P\left(M_{p}, U_{2,3}\right)$ for $M_{p}$ in $\left\{M\left(\mathcal{W}_{3}\right), F_{7}, F_{7}^{*}, A G(3,2)\right\}$.

\section{The 3-COnNeCted Binary $(2,2)$-Uniform Matroids}

In this section we prove Theorem 1.3, and in doing so, complete the determination of the binary $(2,2)$-uniform matroids. We also remark that two of the important matroids of this section, $P_{9}$ and $L_{10}$, arise as graft matroids. A graft [7] is a pair $(G, \gamma)$ where $G$ is a graph and $\gamma$ is a subset of $V(G)$ thought of as the coloured vertices. The associated graft matroid is the vector matroid of the matrix obtained by adjoining the incidence vector of the set $\gamma$ to the vertex-edge incidence matrix of $G$. We follow [3] in using $P_{9}$ to denote the simple binary extension of $M\left(\mathcal{W}_{4}\right)$ represented by the matrix of Figure 2. This is isomorphic to the graft of $\mathcal{W}_{4}$ in which the hub vertex and three of the four rim vertices are coloured. By considering the representation of the matroid $P_{10}$ given in Figure 1 , we see that $P_{10}$ arises as a single-element coextension of $P_{9}$. In fact, it is routine (if tedious) to verify that $P_{10}$ is the 3 -sum of $P_{9}$ and $F_{7}$ across any of the four triangles of $P_{9}$ other than $\{1,4,8\}$ and $\{3,4,7\}$. Up to isomorphism, there are two other simple binary extensions of $M\left(\mathcal{W}_{4}\right)$, namely $M\left(K_{5} \backslash e\right)$ and $M^{*}\left(K_{3,3}\right)$.

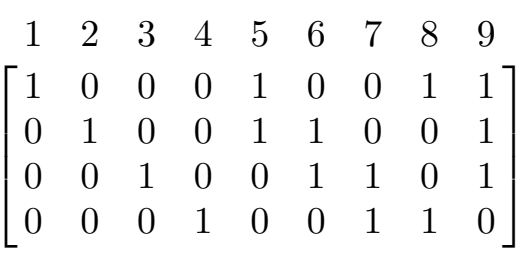

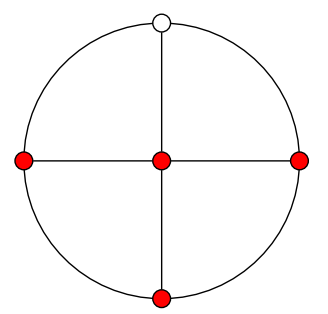

Figure 2. A binary representation of $P_{9}$ and $P_{9}$ as a graft of $\mathcal{W}_{4}$.

In proving Theorem 1.3, we will require the following characterisation of binary matroids with no $M\left(\mathcal{W}_{4}\right)$-minor due to Oxley [3, Theorem 2.1]. Here $Z_{r}$ is the rank- $r$ binary spike with tip $t$ and $y$ is some non-tip element of $Z_{r}$.

Lemma 4.1. Let $M$ be a binary matroid. Then $M$ is 3-connected and has no $M\left(\mathcal{W}_{4}\right)$ minor if and only if

(i) $\quad M \cong Z_{r}, Z_{r}^{*}, Z_{r} \backslash y$, or $Z_{r} \backslash t$ for some $r \geq 3$; or

(ii) $\quad M \cong U_{0,0}, U_{0,1}, U_{1,1}, U_{1,2}, U_{1,3}$, or $U_{2,3}$. 
The flats of the rank- $r$ binary spike are very well behaved and the straightforward proof of the following is omitted.

Lemma 4.2. For $r \geq 3, Z_{r}$ and $Z_{r} \backslash y$ are $(2,2)$-uniform if and only if $r \leq 4$ and $Z_{r} \backslash t$ is $(2,2)$-uniform if and only if $r \leq 5$.

Now consider the rank-5 binary affine geometry $A G(4,2)$. As its rank3 flats are all isomorphic to $U_{3,4}$, this matroid is certainly $(2,2)$-uniform. Viewing $A G(4,2)$ as the deletion of a hyperplane $H$ from the projective geometry $P G(4,2)$, we see that every element of $H$ is in a triangle with two elements of $A G(4,2)$. It follows that any rank-5 binary extension of $A G(4,2)$ must have a rank-3 flat of nullity at least 2 and hence fail to be $(2,2)$-uniform. Furthermore, by Lemma 2.6, $A G(4,2)$ has no binary $(2,2)$ uniform coextensions. Thus, $A G(4,2)$ is a maximal binary $(2,2)$-uniform matroid. The next lemma concerning binary affine matroids will be used in the proof of Theorem 1.3.

Lemma 4.3. Let $M$ be a simple rank-5 binary extension of $M\left(K_{3,3}\right)$. Then $M$ is (2,2)-uniform if and only if $M$ is affine.

Proof. If $M$ is a simple rank-5 binary affine matroid, then it is a restriction of $A G(4,2)$ and thus is $(2,2)$-uniform. For the other direction, let $M$ be a simple rank-5 binary extension of $M\left(K_{3,3}\right)$ that is $(2,2)$-uniform. By uniqueness of binary representation, $M$ may be represented by a binary matrix whose first nine columns are the representation of $M\left(K_{3,3}\right)$ given in Figure 3. Let $e$ label an extension column. It is easily seen that if the last entry of column $e$ is zero, then $e$ is in a triangle with two elements of $M\left(K_{3,3}\right)$. But every pair of elements of $M\left(K_{3,3}\right)$ are in a circuit of size four. Thus, if column $e$ ends in zero, then $e$ is in a rank-3 flat of $M$ of nullity at least 2. This is a contradiction to the $(2,2)$-uniform property. We conclude that every extension column ends in 1 and that, consequently, $M$ is affine.

$\left.\begin{array}{lllllllll}1 & 2 & 3 & 4 & 5 & 6 & 7 & 8 & 9 \\ 1 & 0 & 0 & 0 & 0 & 1 & 0 & 0 & 1 \\ 0 & 1 & 0 & 0 & 0 & 1 & 1 & 0 & 0 \\ 0 & 0 & 1 & 0 & 0 & 0 & 1 & 1 & 0 \\ 0 & 0 & 0 & 1 & 0 & 0 & 0 & 1 & 1 \\ 1 & 1 & 1 & 1 & 1 & 1 & 1 & 1 & 1\end{array}\right]$

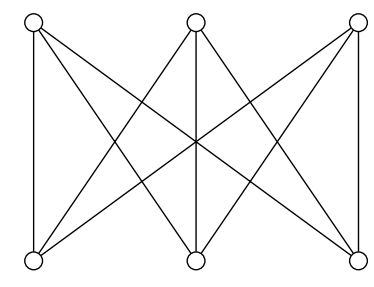

FiguRE 3. Binary and graphic representations for $M\left(K_{3,3}\right)$.

Two of the four non-isomorphic simple rank-5 binary single-element extensions of $M\left(K_{3,3}\right)$ are affine. These are the well-known regular matroid $R_{10}$ and a matroid that we name $L_{10}$, a representation for which is given in Figure 4. In [7], $R_{10}$ is identified as the graft matroid of $K_{3,3}$ in which every 


\begin{tabular}{|c|c|c|c|c|c|c|c|c|}
\hline 1 & 2 & 3 & 4 & 5 & 6 & 7 & 8 & \\
\hline$[1$ & 0 & 0 & 0 & 0 & 1 & 0 & 0 & \\
\hline 0 & 1 & 0 & 0 & 0 & 1 & 1 & 0 & \\
\hline 0 & 0 & 1 & 0 & 0 & 0 & 1 & 1 & ) \\
\hline 0 & 0 & 0 & 1 & 0 & 0 & 0 & 1 & \\
\hline 1 & 1 & 1 & 1 & 1 & 1 & 1 & 1 & \\
\hline
\end{tabular}

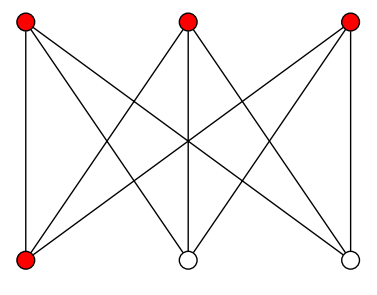

FiguRE 4. A binary representation of $L_{10}$ and $L_{10}$ as a graft of $K_{3,3}$.

vertex is coloured. We remark here that $L_{10}$ is the graft matroid of $K_{3,3}$ in which all but two vertices, both in the same partition, are coloured.

In our final step before proving Theorem 1.3, we determine the binary (2,2)-uniform coextensions of $M\left(K_{5} \backslash e\right)$ and $P_{9}$; geometric representations of which are given in Figure 8.

Lemma 4.4. The sets of non-isomorphic binary $(2,2)$-uniform coextensions of $M\left(K_{5} \backslash e\right)$ and $P_{9}$, respectively, are $\left\{L_{10}\right\}$ and $\left\{P_{10}, L_{10}\right\}$.

Proof. Let $M$ be a binary (2,2)-uniform matroid with a subset $X \subseteq E(M)$ such that $M / X \cong N$ for $N$ in $\left\{M\left(K_{5} \backslash e\right), P_{9}\right\}$. By uniqueness of binary representation, we may assume that $M / X$ is represented by the binary matrix $A$ given in Figure 5, where $\alpha \in\{0,1\}$ depends on $N$.

\begin{tabular}{|c|c|c|c|c|c|c|c|c|}
\hline$e_{1}$ & $e_{2}$ & $e_{3}$ & $e_{4}$ & $e_{5}$ & $e_{6}$ & $e_{7}$ & $e_{8}$ & $e_{9}$ \\
\hline 1 & 0 & 0 & 0 & 1 & 0 & 0 & 1 & 1 \\
\hline 0 & 1 & 0 & 0 & 1 & 1 & 0 & 0 & $\alpha$ \\
\hline 0 & 0 & 1 & 0 & 0 & 1 & 1 & 0 & 1 \\
\hline 0 & 0 & 0 & 1 & 0 & 0 & 1 & 1 & 0 \\
\hline
\end{tabular}

Figure 5. Matrix $A . M[A]$ is isomorphic to $M\left(K_{5} \backslash e\right)$ when $\alpha=0$ and $P_{9}$ when $\alpha=1$, respectively.

The set $H=\left\{e_{1}, e_{2}, e_{3}, e_{5}, e_{6}, e_{9}\right\}$ is a hyperplane of $M[A]$ regardless of $\alpha$. As $M$ is (2,2)-uniform, it follows that $H \cup X$ is a hyperplane of $M$ of nullity 3. Moreover, $M \mid H \cup X$ is $(1,2)$-uniform and $(M \mid H \cup X)^{*}$ is $(2,1)$-uniform by duality. Thus, by Lemma 2.2, $(M \mid H \cup X)^{*}$ is a rank-3 simple matroid with $|X|+6$ elements. It follows that $|X|=1$. By appropriate row operations, one then sees that $M$ may be represented by the $5 \times 10$ binary matrix $B$ as given in Figure 6. It remains to determine the coefficients $\beta_{5}, \ldots, \beta_{9}$.

A representation for $(M \mid H \cup x)^{*}$ is given in Figure 7. As this must be simple, we deduce that $\beta_{5}=\beta_{6}=1$ and $\beta_{9}=1-\alpha$. To determine $\beta_{7}$ and $\beta_{8}$, we consider the hyperplane $H^{\prime}=\operatorname{cl}_{M}\left(\left\{e_{1}, e_{3}, e_{4}\right\}\right)$ of $M[A]$. If $\alpha=0$, then the hyperplane $H^{\prime} \cup x$ of $M$ contains $e_{9}$ and by an identical argument to before, $\beta_{7}=\beta_{8}=1$. We conclude that if $N \cong M\left(K_{5} \backslash e\right)$, then $M \cong L_{10}$. 


\begin{tabular}{|c|c|c|c|c|c|c|c|c|c|}
\hline$e_{1}$ & $e_{2}$ & $e_{3}$ & $e_{4}$ & $e_{5}$ & $e_{6}$ & $e_{7}$ & $e_{8}$ & $e_{9}$ & $x$ \\
\hline 1 & 0 & 0 & 0 & 1 & 0 & 0 & 1 & 1 & 07 \\
\hline 0 & 1 & 0 & 0 & 1 & 1 & 0 & 0 & $\alpha$ & 0 \\
\hline 0 & 0 & 1 & 0 & 0 & 1 & 1 & 0 & 1 & 0 \\
\hline 0 & 0 & 0 & 1 & 0 & 0 & 1 & 1 & 0 & 0 \\
\hline 0 & 0 & 0 & 0 & $\beta_{5}$ & $\beta_{6}$ & $\beta_{7}$ & $\beta_{8}$ & $\beta_{9}$ & 1 \\
\hline
\end{tabular}

Figure 6. Matrix $B . M[B] / x$ is isomorphic to $M\left(K_{5} \backslash e\right)$ when $\alpha=0$ and $P_{9}$ when $\alpha=1$, respectively.

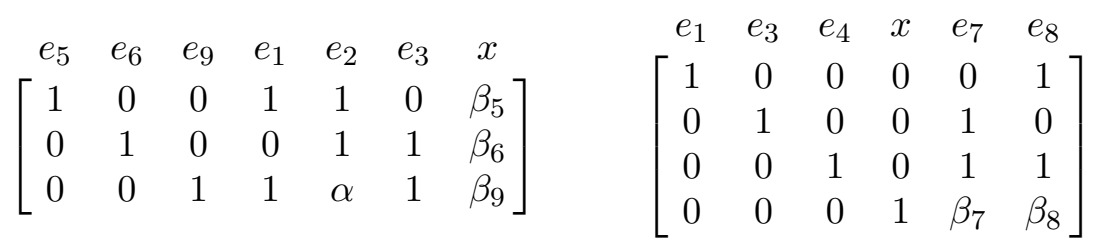

FiguRE 7. Matrices representing $(M \mid H \cup x)^{*}$ and $M \mid H^{\prime} \cup x$.

Otherwise $N \cong P_{9}, \alpha=1$ and $H^{\prime}=\left\{e_{1}, e_{3}, e_{4}, e_{7}, e_{8}\right\}$. Then $M \mid H^{\prime} \cup x$ is represented by the rank- 4 matrix of Figure 7 . As this matroid must be $(1,2)$-uniform, it follows that either $\beta_{7}=\beta_{8}=1$, in which case $M \cong L_{10}$, or precisely one of $\left\{\beta_{7}, \beta_{8}\right\}$ is zero, in which case, $M \cong P_{10}$.
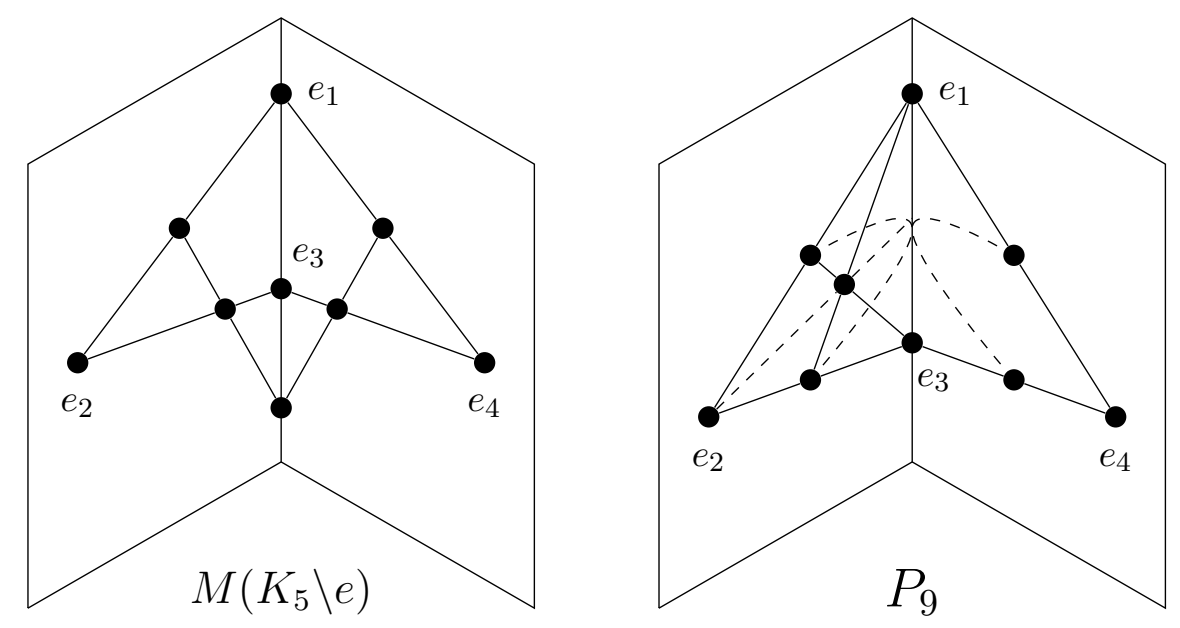

Figure 8. Geometric representations of $M\left(K_{5} \backslash e\right)$ and $P_{9}$.

We now conclude the paper by proving Theorem 1.3.

Proof of Theorem 1.3. We first observe that a matroid is a binary 3-connected $(2,2)$-uniform matroid if and only if its dual is also. In particular, both $A G(4,2)$ and $A G(4,2)^{*}$ are minor-maximal such matroids. To complete our 
list, let $M$ be a minor-maximal binary 3 -connected $(2,2)$-uniform matroid. If $r(M) \leq 4$, or $r^{*}(M) \leq 4$, then $M$ is a minor of either $A G(4,2)$ or $A G(4,2)^{*}$, a contradiction to maximality. Thus, $r(M), r^{*}(M) \geq 5$. Switching to the dual if necessary, we may then assume by Lemma 2.6 that $r(M)=5$.

If $M$ has no $M\left(\mathcal{W}_{4}\right)$ minor, then by Lemma $4.1, M$ is isomorphic to one of $Z_{r}, Z_{r}^{*}, Z_{r} \backslash t, Z_{r} \backslash y$ for some $r \geq 3$ and, by Lemma $4.2, M \cong Z_{5} \backslash t$. Otherwise, we may assume that $M$ does possess an $M\left(\mathcal{W}_{4}\right)$-minor. Then, as $r(M)=5$, $M$ is an extension of a single-element coextension $N$ of $M\left(\mathcal{W}_{4}\right)$. As $M\left(\mathcal{W}_{4}\right)$ is self-dual, the matroid $N^{*}$ is a binary $(2,2)$-uniform single-element extension of $M\left(\mathcal{W}_{4}\right)$. These are just the simple binary extensions of $M\left(\mathcal{W}_{4}\right)$, namely $M\left(K_{5} \backslash e\right), P_{9}$ and $M^{*}\left(K_{3,3}\right)$. Thus, $N \in\left\{M^{*}\left(K_{5} \backslash e\right), P_{9}^{*}, M\left(K_{3,3}\right)\right\}$. If $N \cong M\left(K_{3,3}\right)$, then by Lemma $4.3, M$ must be affine and thus, by maximality, $M \cong A G(4,2)$. Otherwise, $N \in\left\{M^{*}\left(K_{5} \backslash e\right), P_{9}^{*}\right\}$, in which case, by the dual of Lemma 4.4, $M$ is isomorphic to either $P_{10}$ or $L_{10}^{*}$. But, as $L_{10}$ is affine, $L_{10}^{*}$ is a minor of $A G(4,2)^{*}$. We conclude by maximality that, in this case, $M \cong P_{10}$. The theorem then follows by duality.

\section{ACKNOWLEDGEMENTS}

The author thanks Professors Charles Semple and James Oxley for reading the early drafts of this paper and for their invaluable suggestions towards improving the exposition.

\section{REFERENCES}

[1] Acketa, D., On binary paving matroids, Discrete Math. 70 (1988), 109-110.

[2] Geelen, J., Gerards, B., Whittle, G., Solving Rota's conjecture, Notices Amer. Math. Soc., 61 (2014), 736-743.

[3] Oxley, J.G., The binary matroids with no 4-wheel minor., Trans. Amer. Math. Soc. 301 (1987), 63-75

[4] Oxley, J.G, Ternary paving matroids, Discrete Math. 91 (1991), 77-86.

[5] Oxley, J., Matroid Theory, Second edition, Oxford University Press, New York, 2011.

[6] Rajpal, S., On binary k-paving matroids and Reed-Muller codes, Discrete Math. 190 (1998), 191-200.

[7] Seymour, P.D., Decomposition of regular matroids, J. Combin. Theory Ser. B 28 (1980), 305-359.

School of Mathematics and Statistics, University of Canterbury, Christchurch, NEW ZEALAND

Email address: george.drummond@pg. canterbury.ac.nz 